\title{
Dupilumab Reduces Blood, Urine, and Nasal Biomarkers of Type 2 Inflammation in Patients With Chronic Rhinosinusitis With Nasal Polyps in the Phase 3 SINUS-52 Trial
}

Claus Bachert ${ }^{1,2}$, Seong H. Cho ${ }^{3}$, Tanya M. Laidlaw ${ }^{4}$, Brian N. Swanson ${ }^{5}$, Sivan Harel ${ }^{6}$, Leda P. Mannent ${ }^{7}$, Nikhil Amin ${ }^{6}$, Alexandre Jagerschmidt ${ }^{7}$ 'Ghent University, Ghent, Belgium; '2Karolinska Institutet, Stockholm, Sweden; ${ }^{3}$ University of South Florida, Tampa, FL, USA; “Brigham and Women's Hospital, Boston, MA, USA; ${ }^{5}$ Sanofi, Bridgewater, NJ, USA;

\section{BACKGROUND}

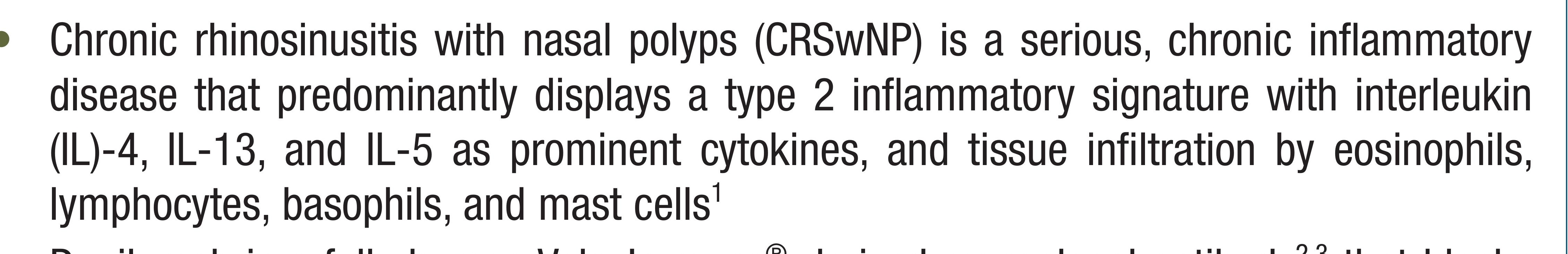

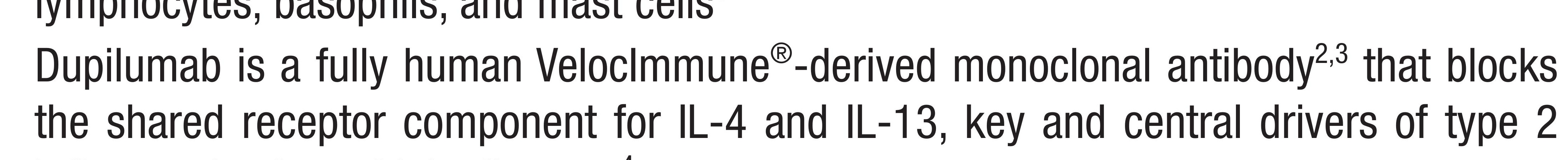
inflammation in multiple diseases"

Dupilumab is approved in the USA as an add-on treatment in adult patients with
inadequately controled CSSWNP.; dupilumab is also approved in certain patients with

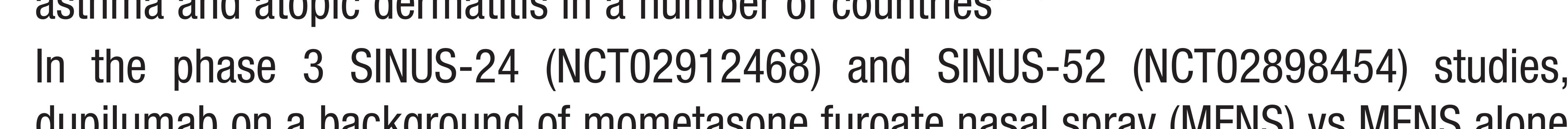

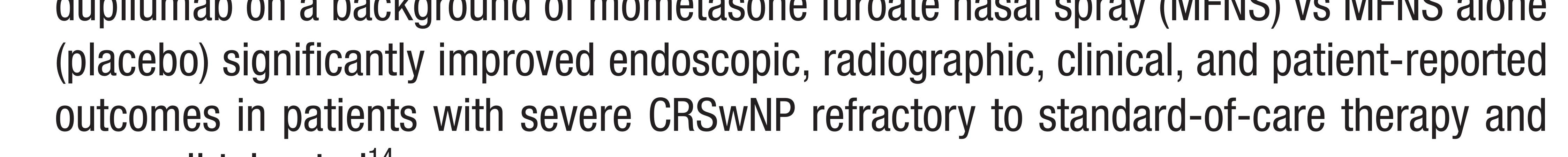

AIM

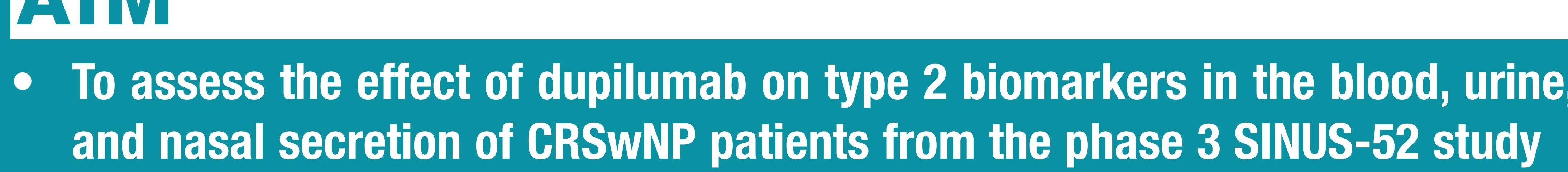
METHODS
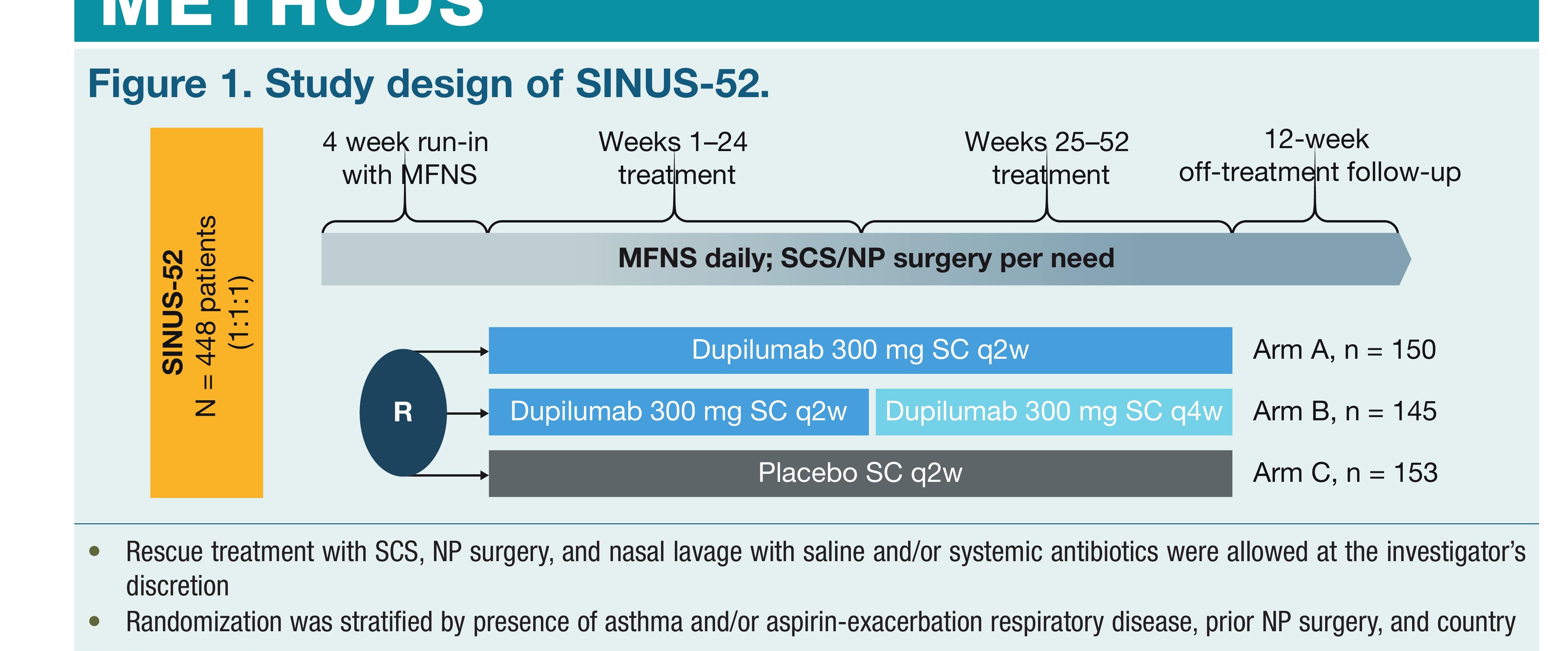

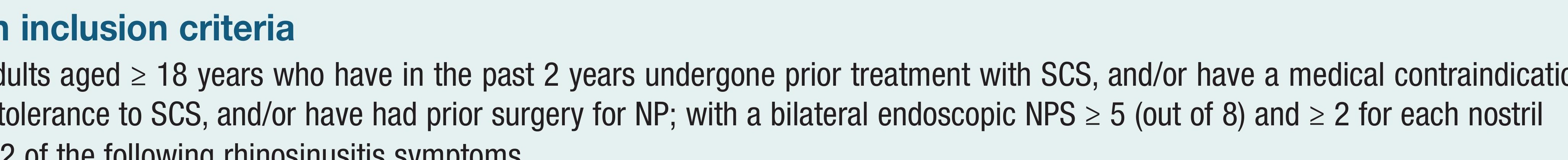

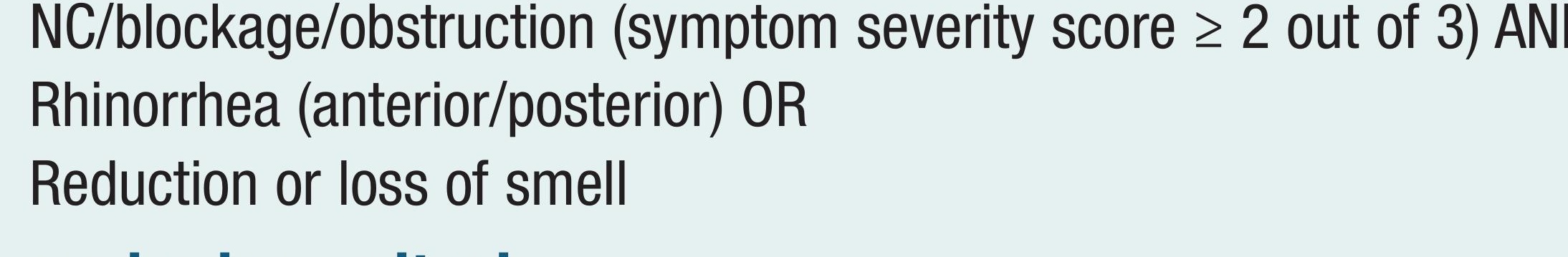

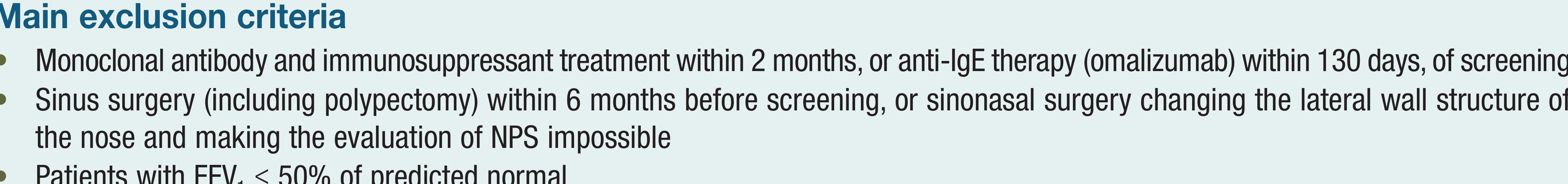

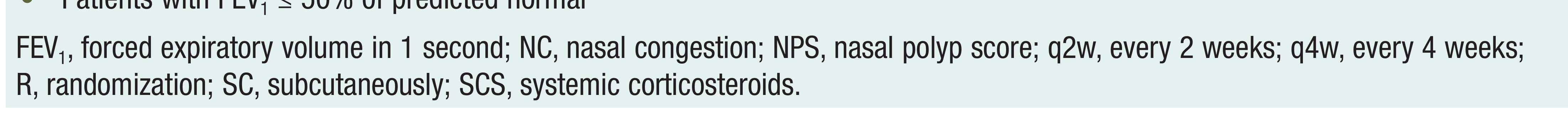
Study assessments

- On-treatment biomarkers in blood, urine, and nasal secretion were
placebo and pooled dupilumab q2w groups in the safety population

\section{RESULTS}

Figure 2. Dupilumab reduces biomarkers of type 2 inflammation in patients with CRSWNP. Blood: (A) total IgE; (B) periostin; (C) TARC; (D) eotaxin-3; (E) eosinophils. Nasal secretion: (F) total lgE; (G) periostin; (H) LL-5; (I) eotaxin-3.
()) Urinary LTE4.
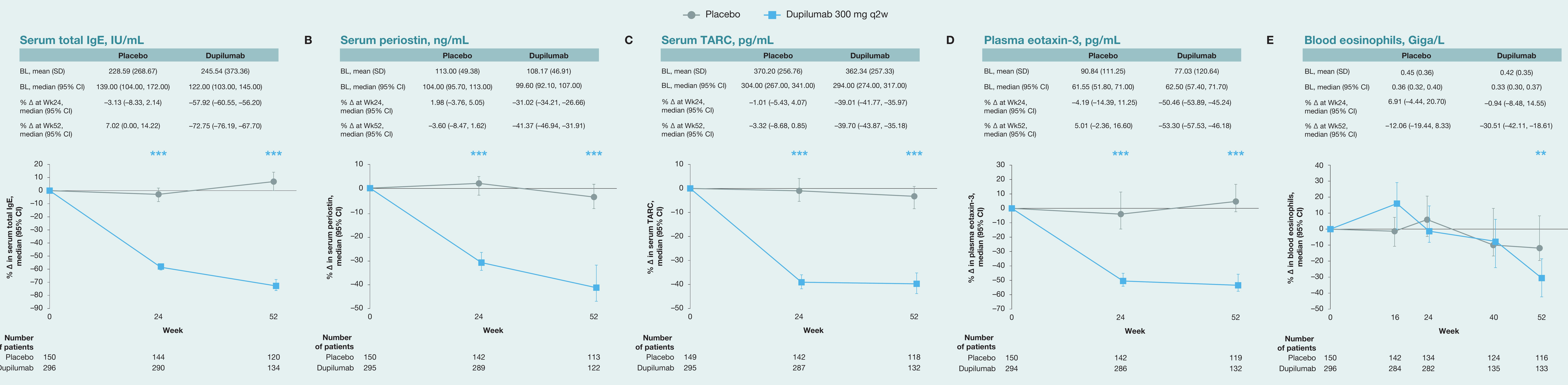

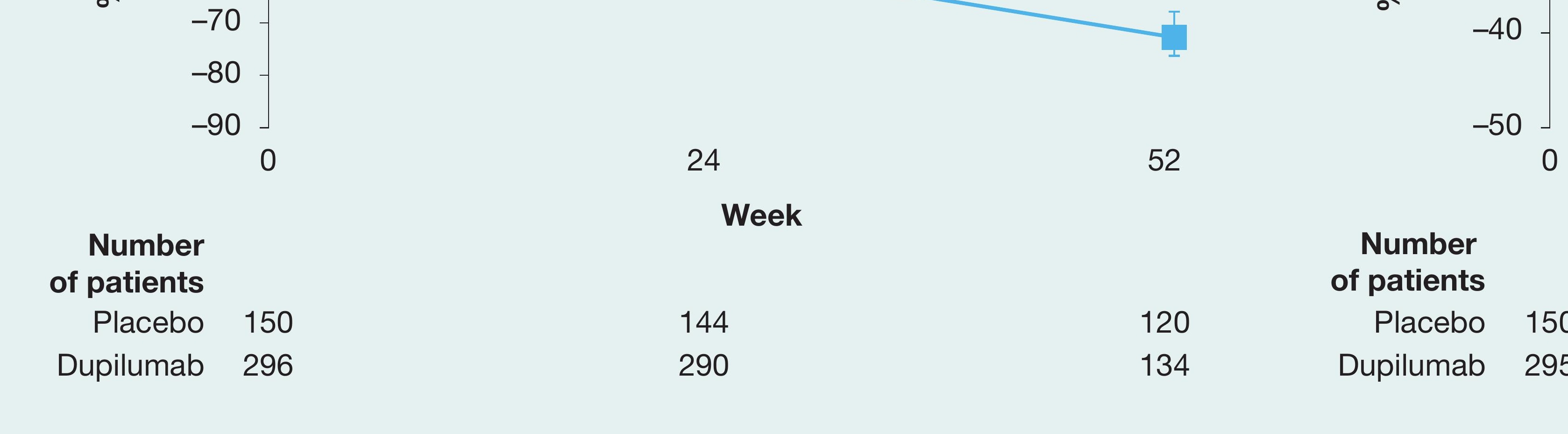
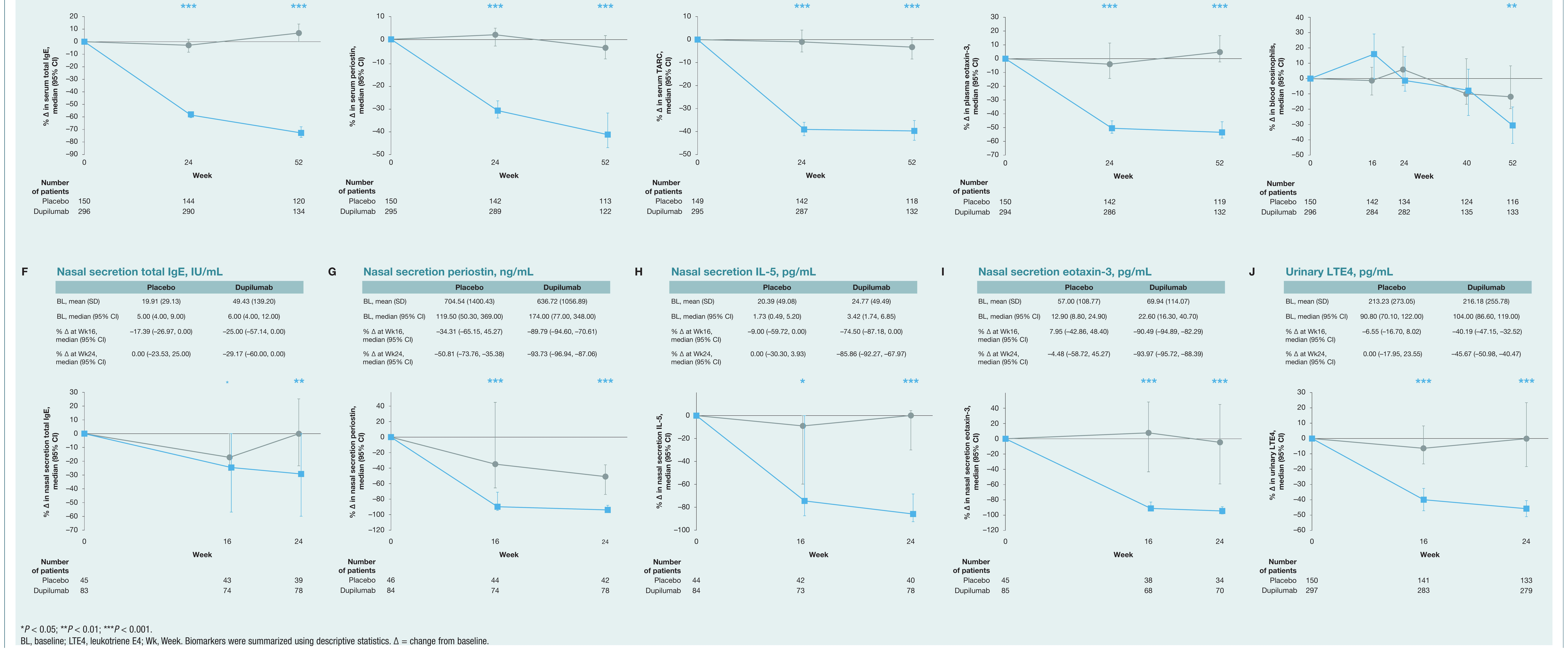

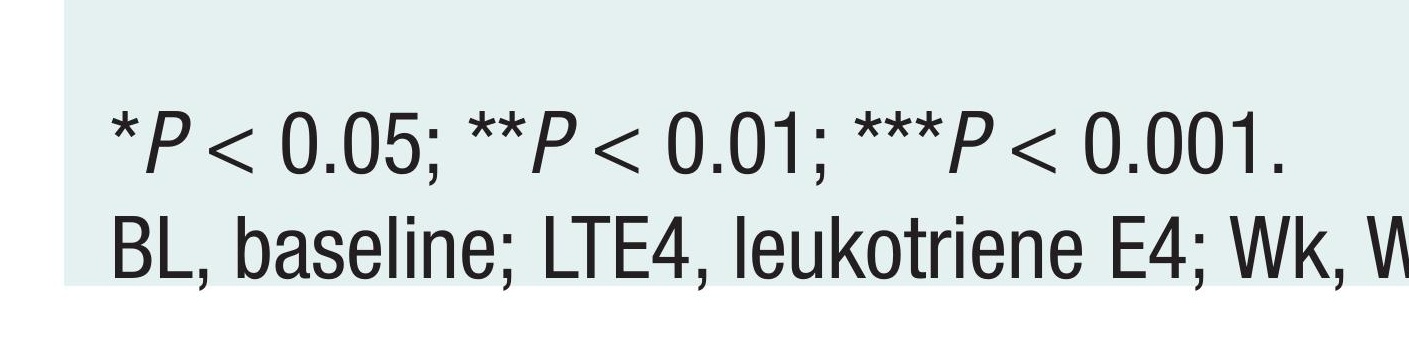

(94)

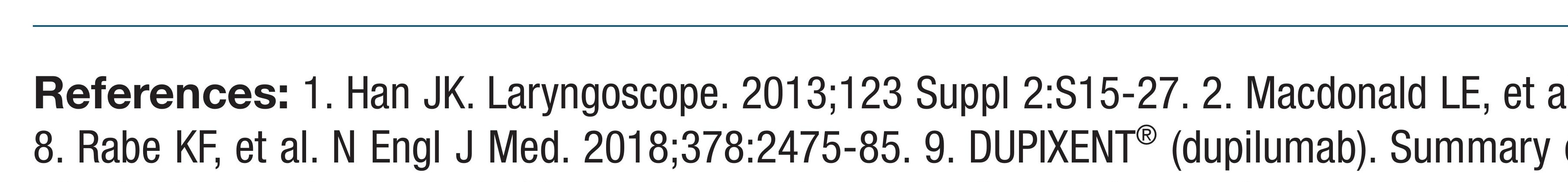

